\title{
Conception of 4-Component Architecture of Information Systems on Example of Artificial Neural Networks
}

\author{
Dmitriy Gakh \\ Institute of Control Systems, \\ Bakhtiyar Vahabzadeh str. 68 , \\ AZ1141, Baku, Azerbaijan \\ Email: dgakh@sinam.net
}

\begin{abstract}
Nowadays Information Systems (IS) become more and more distributed, complex, and heterogeneous. Such nature of IS make them or their components a Black Box. Although classical software operates according understandable logic, modern complex software often shows non-determinism in its operation. Artificial Intelligence (AI) based on Artificial Neural Networks (ANN) is an example of such systems.

This paper considers IS architecture consisting of 4 components, one of which represents non-determinism as an "Machine Intuition". The architecture is derived from 3-tier computer architecture and based on psychological findings. This approach allowed building a simple and user/developer friendly model.

Practical value of the architecture is concluded in ability to better understand, design, and develop the IS containing units with non-deterministic behavior, deal with AI overfitting, underfitting, and threat problems. Architecture and principles represented in this paper can be applied not only to AI/ANN but different IS types.
\end{abstract}

\section{INTRODUCTION}

A RTIFICIAL Intelligence (AI) is one of the most intensively developing technology. Design of simple AI systems is concluded in composing the Artificial Neural Network (ANN) layout (in this article, unless otherwise stated, the abbreviation AI refers to AI based on ANN). However, at this stage there is no clear understanding of how the ANN will operate. This fact demonstrates a difference of AI development and that of classical software. So, while classical software is a series of commands and its behavior is deterministic, AI introduces some kind of non-deterministic behavior. Another example of non-deterministic calculations is quantum computing (QC / A QC is not a deterministic machine; in other words, there is no single solution for which any other result would be an error [1]). To simplify the readability, the further text will discuss AI, but many statements can be also applied to other technologies. The article mentions AI non-deterministic behavior and units providing "Machine Intuition" (there is also a term "Artificial Intuition").

Since ANN are originated from observation of real life processes, life simulation solutions are very interesting examples where results of their operations can be compared to life. Genetic and Machine Learning Algorithms should be also considered because in most cases they provide non-deterministic results. A very interesting example is the life simulation where wolfs preferred suicide over eating sheep [2]. There are also other examples showing unintended consequences of Black Boxes [2-5]. Complex systems are intrinsically hazardous systems. Complex systems contain changing mixtures of failures latent within them, change introduces new forms of failure, human operators have dual roles: as producers and defenders against failure, human practitioners are the adaptable elements of complex systems, etc. [6].

The AI Failures Incident Database provides a publicly accessible view of AI failures [7,8]. The classification schema detailing AI failures has been developed [7, 9]. The methods of avoiding AI failures that provides a balance between being excessively rigid (which would make its use difficult and brittle) and overly subjective (which would render the framework useless) have been elaborated [7].

Many problems presented by a super intelligence resemble exercises in international diplomacy more than computer software challenges; for instance, the value alignment problem of aligning AI values with humans'. Failure is defined as 'the nonperformance or inability of the system or component to perform its expected function for a specified time under specified environmental conditions'. Intelligence definitions converge towards the idea that it 'measures an agent's ability to achieve goals in a wide range of environments' [9]. These definitions imply such requirements as specified time and specified environmental conditions. However, actual AI solutions can be applied for undefined period of time and in unpredictable environment. Thus, failures of AI systems are non-deterministic nature. We can say here about something like "Machine intuition" instead of failures. We can as well say that there is a kind of "Machine intuition" failures. "Machine Intuition" can be presented in computer architecture as a separate specific component.

Architecture of solution is an important conception that to be formulated at the very beginning of the development. Although there are papers describing AI or QC solutions architecture, this description is specific and quite complex (One example is AI Infrastructure Reference Architecture from IBM [10]. Other examples are described in [11, 12] representing QC hardware architectures). Literature analysis shows that there is lack of papers describing general architecture of AI solutions reflecting their generic features, the most important of which can be non-determinism (at least in 
comparison with classic software). Absence of such literature is a gap between understanding of AI solutions and well known classical (3-tier or N-tier) solutions. As a result it prevents smooth integration of AI and the classical solutions (implementation of AI in the classical solutions become popular method of software upgrade).

This paper considers simple 4-component architecture of IS solution derived from 3-tier architecture. This approach allows describing AI architecture as understandable for the classical programmers (those who write software on base of 3-tier or N-tier architectures) and integrate AI with the existing solution more smoothly. The research considers psychological findings that make the model closer to the human thinking and better align AI values with humans. Some phenomena that previously considered as failures can now be considered as behavior of "Machine Intuition". Introduction of computer architecture that simplifies development of software with complex unpredictable behavior is the main motivation to carry out this research. The main contribution of the article is rising the question about presence in modern IS components with non-deterministic behavior and necessity to study it. 4-component IS architecture and some problems that can be solved by its means are presented.

\section{Background and Research Questions}

AI has its own advantages and disadvantages. It differs from classical determined software. One requirement of classical software is providing determined output for determined input. This requirement is assured by tests. However, size and complexity of input data grow exponentially and it leads to impossibility to test the software for each case. This fact in its turn leads to non-determinism when input data or IS architecture become large and complex. Software Development methodologies do not reflect this non-determinism explicitly. However, it should be mentioned that quality assurance methods are effective in many cases. Nevertheless, these methods regard this non-deterministic behavior as a disadvantage. As a result, this can reduce the flexibility of AI solutions.

Software Quality itself is not a deterministic conception. The definitions of "quality" shows that it is not an objective index. Software Quality Models introduce metrics to make quality measurable. Due to interrelation of quality attributes a trade-offs must take place. There are also stakeholder's expectations that needs trade-offs between them [13].

In practice, a gap exists between abstract quality definitions provided in common quality taxonomies, such as ISO 25010 , and concrete quality assessment techniques and measurements $[14,15]$. Company-specific quality models are widely used. Quality models typically are adapted. ISO standards are not well accepted. Quality model users are moderately satisfied with their models [16].

Architecture of IS solutions impacts its quality. Analyses of literature demonstrates lack of description of AI architectures that will be simple and close to the classic software architectures. Because classic software architectures do not contain a component reflecting non-deterministic behavior, designers cannot pay significant attention to this IS character. Introduction of separate component reflecting "Machine Intuition" allowed designing IS with possible non-deterministic behavior in mind. Thus, it makes sense to design an architecture that takes this feature into account.

Meanwhile, AI originated from discovery of human brain's neural structure. This fact allowed to look for application of psychological findings to the AI solutions. Design of AI solution architecture on base of psychological findings has two benefits:

- Basic primitive rules that are true for the human brain most likely will be true for AI, because AI is originated from human brain's neural structures (proving of this hypothesis is out of scope of current research);

- A solution based on psychological findings will be likely close to human understanding (there are papers about research of psychological approaches to the software development processes).

\section{A. Three-Tier Architecture}

Three-tier architecture was developed by John J. Donovan in Open Environment Corporation (OEC), a tools company he founded in Cambridge, Massachusetts [17-19]. Fowler describes three principal layers of computer architecture as the following [20]:

1. Data Source - Databases, messaging systems, transaction managers, etc.;

2. Domain - Logic that is the real point of the system;

3. Presentation - Provision of services, display of information, user interface, HTTP requests, command-line invocations, batch API.

Three-tier computer architecture is the classical architecture that can be used to design complex IS or development of small applications. Division of IS into three tiers allows not only to simplify the design, but also distribute tasks between different developers. So, for example, the data tier can be developed and maintained by database specialists, logic tier developed by programmers, and presentation designed by user interface designers.

\section{B. Literature about Psychology in Computer Science}

Computer science can be applied to many aspects of human life. One of the them is psychology. This article does not consider application of computers in psychology. It rather considers application of psychology in computer science.

Proper design of interfaces between humans and machines humans wish to control requires cooperation of engineers and psychologists. Such cooperation allows dealing with so called "Human Factors" [21]. Considering psychological issues in human-computer interaction is not a new approach [22]. References to the synthesis of psychological knowledge and computer science are also mentioned in later sources [23]. In his research Prabhaker Panditi concluded that Software Engineering should consider the latest scientific discoveries in psychology, social psychology and be- 
havioral economics. There is a need to conduct experiments to identify how the psychological discoveries apply to various phases, processes and practices in Software Engineering [24]. Kam Hou VAT considers so called "Software Psychology" as the domain of human behavior study in software engineering [25].

\section{McWhinney's Realities}

McWhinney's model is selected due to its simplicity and prove in practice (Young and Kovalev [26, 27]). Three-tier computer architecture can be mapped to 3 of 4 of his realities. Additional "Machine Intuition" component can be mapped to the 4th McWhinney's reality. This simple approach allowed building an effective computer architecture model.

Will McWhinney proposes to consider phenomena through a prism of four realities. He has drawn the coordinate system where axis $\mathrm{X}$ represented monistic - pluralistic quality and axis Y represented free will - determined quality. By this way, quarter "monistic + determined" represents unitary (U) reality, quarter "pluralistic + determined" represents sensory (SE) reality, quarter "pluralistic + free will" represents social (SO) reality, and quarter "monistic + free will" represents social (M) reality (See Fig. 1, Fig. 2) [28, 29]. Young and Kovalev have expanded this model and applied it to solve psychological problems [26, 27].

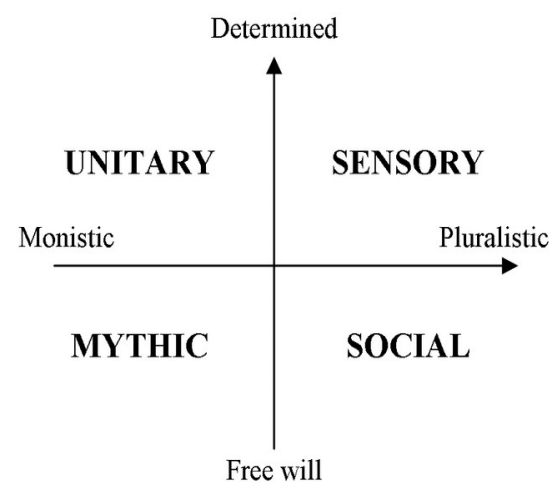

Fig 1. McWhinney's Realities

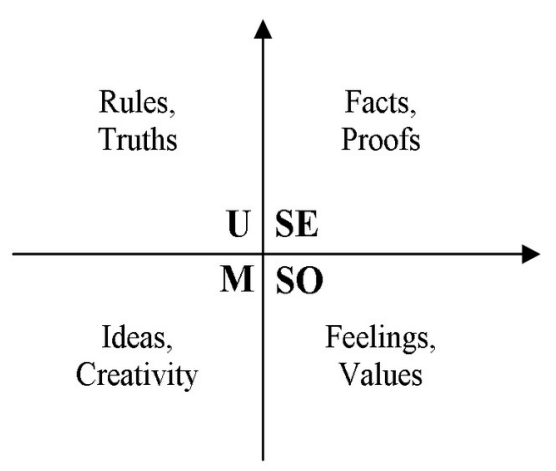

Fig 2. Characteristics of McWhinney's Realities
Phenomena situated in the unitary reality are characterized by rules and truths (See Fig. 2). They represent something stable and unchangeable. Phenomena situated in the sensory reality are characterized by facts and proofs. They represent something useful and profitable. Phenomena situated in the social reality are characterized by feelings and values. They represent something pleasant and comfortable. Phenomena situated in the mythic reality are characterized by ideas and creativity. They represent something synergetic and intuitive.

\section{Research Questions}

Building a 4-component IS architecture on base of 3-tier architecture is not a trivial problem. We cannot say that we just add 4th tier into architecture. Even the structure of the architecture model is not complex, the model changes dramatically. So, the research questions to be answered are:

RQ1: What is the 4-component IS architecture and what is its structure?

RQ2: How the 4-component IS architecture relates to 3tier architecture?

RQ3: Which advantages/disadvantages does the 4-component IS architecture have?

\section{E. Research Methodology}

This research is based on the following main steps:

1. Select appropriate psychological model (McWhinney's realities in this research;

2. Juxtapose components of 3-tier architecture with the McWhinney's realities;

3. Juxtapose AI component with the McWhinney's realities;

4. Build the 4-component IS architecture model on base of identified patterns;

5. Prove the model theoretically.

Scope of this research allows presenting only basic findings and perform simple theoretical proof. Full theoretical proof of the model requires studying many implicit factors and interrelations. Practical proof of the 4-component IS architecture requires attempts to implement it in actual projects. Thus, this research implies in descriptive design and is based on literature analyses, in 28-years author's experience in software development, in experience in psychological and AI studies.

According to abstraction hierarchy [30] this paper describes the following phenomena:

- Theory. Presented 4-component architecture is a theory;

- Concepts. The concepts are presented by tiers of 3tier architecture, realities of McWhinney's model, and components of the 4-component architecture;

- Indicators. Indicators are identified patterns of relations and interrelations of Software Components and realities of McWhinney's model;

- Variables. Variables are presented by components of the models (4-component AI architecture and McWhinney's 
model should be considered together until the theory matures).

- Values. Values are actual software modules, pieces of code, and even hardware devices.

\section{FINDINGS}

IS non-determined behavior occupies more and more spaciousness. Quality Assurance is aimed to mitigate and eliminate its negative effects. However, number of Quality Assurance methods are non-determined themselves because they are subjective.

Synthesis of computer science and psychology is not a new conception. Psychology is considered mainly for human-computer or human-robot interaction. Behavioral psychology is the main direction of psychology discussed in previous studies. Human factors can be considered as psychological phenomena.

Model of McWhinney's realities shows the 4 types of human vision of the world. Its practical value is proven in management and psychotherapy and has a simple basic structure.

As a result of considering of 3-tier architecture through the prism of McWhinney's realities the following parallels can be drawn (See Fig. 3):

1. Data Source relates to unitary reality because it represents rules (relations, constraints...) and truths (data). The statement "Deterministic systems of truths, assumptions, and propositions. Logics, morality, and spiritual oneness." [28] most likely relates to the data source;

2. Domain relates to sensory reality because facts and proofs can be provided by the logic. The statement "Raw characteristics and atomistic objects are derived from the senses. Empiricism." [28] most likely relates to the data processing by the functions. These functions in their turn represent the programming logic;

3. Presentation relates to social reality because feelings and values are the result of presentation abilities. Statement "Emotions and group values associated with distinct individuals and groups. Ethics and human relations." [28] most likely relates to the presentation. In other words, presentation of a computer system determines emotions of its users and their group values.

\section{Discussion of Findings}

ANN are underlying structures of AI systems in most cases. AI solutions in their turn show non-deterministic behavior to a greater extent. In other words, they show "Machine Intuition". So, ANN are the best example to discuss 4component architecture. Other types of solutions can be discussed in the same manner. Indeed, solutions with determined behavior can be presented without "Machine Intuition" component. Bugs and errors can be considered as belonging to "Machine Intuition" component where they can be considered as "wrong decision of the intuition". This statement is a subject for further discussions. Complex IS could be considered as set of interconnected components

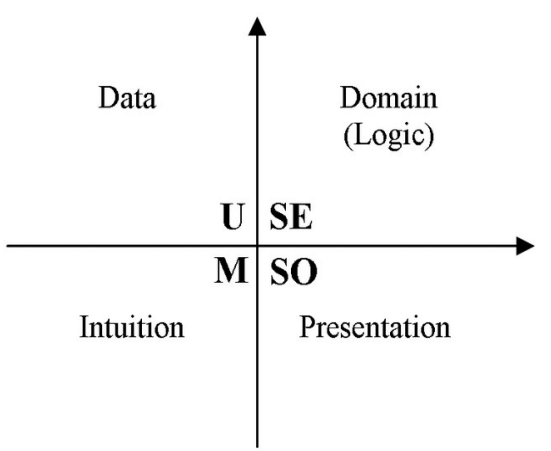

Fig 3. Components of the Model in McWhinney's Realities

forming network structures like ANN where each component can be considered as an artificial neuron. Testing and debugging of such systems could be considered as supervised Machine Learning (ML). Non-deterministic behavior of such systems could have the same origin as ANN. These facts confirm that although 4-component architecture is build on example of ANN, it can be applied for IS of different types.

ANN provide generalization of many data at a time. AI systems give a result with some uncertainty. Even if the system gives 10000 results with confidence $99.99 \%$, there is no assurance that the next result will be of such high confidence. On the one hand, the deviation can be considered as an error, on the other hand it can be considered as an AI's intuition, idea, or creativity. Most often AI systems are too complex to make any assumption about their operation and represent Black Box. They require learning and testing after building. All these features of AI systems show that according to McWhinney's model it belongs to the mythic reality.

One can say that AI relates to the sensory reality. But AI systems are built as one whole construction processing large data entirely in one operation (by one call). Sensory reality is specified by "atomistic objects" [28] that corresponds more to number of functions processing number of data by number of operations (iterations, calls). One can also say that AI could be assigned to unitary reality because it is represented by the structure of neural network. But this structure is active and cannot be considered as the static data. Some kind of uncertainty does not allow considering AI as an element of unitary reality.

\section{A. Four Components vs. Three Tiers}

Three tier model contains 3 computer components connected sequently. Thus, these components can be called "tiers". But addition of AI as the fourth component leads to change of the system orderly structure then the components cannot be named "tiers" any more. The new structure is presented by Fig. 4. It should be marked that AI component within the 4-component architecture is presented as "Intuition". It is because the whole model can represent an AI solution. Indeed, an AI solution contains data, presentation, 
and logic (this is also described in the text below). To follow technical language, one can say that "Machine Intuition" is better term for naming computer components relating to the 4th component. But "Intuition" is laconic and simple to use in text and diagrams.

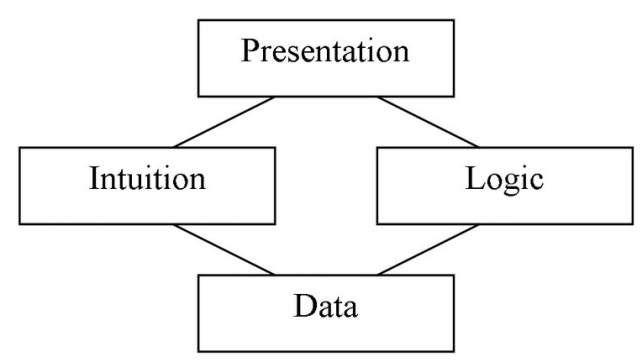

Fig 4. Four-Component Architecture

This layout is the most correct one by the following reasons:

- It could be considered as derived from 3-tier model where tier "Domain" is divided into two components "Logic" and "Intuition";

- According to McWhinney's realities "Data" locates beside "Logic" and "Intuition", "Logic" locates beside "Data" and "Presentation", "Presentation" locates beside "Logic" and "Intuition", and "Intuition" locates beside "Data" and "Presentation". Such closeness determines the connections between the components;

- AI systems can be even represented by 3-tier model where AI locates in tier "Domain" (but after introduction the 4-component model, use of 3-tier model for IS could be considered as deprecated).

McWhinney's model considers all relations between the realities. According to this principle, "Presentation" can be connected to "Data" and "Intuition" can be connected to "Logic" (See Fig. 5). Considering the "Presentation-Data" connection is not interested in current research because it is equal to corresponding connection between tiers in 3-tier model. But considering the "Logic-Intuition" connection should be discussed within this research because "Intuition" is a new component (relating to 3-tier model). At the same time this connection cannot be implemented easily because "Logic" relates to determined conception while "Intuition" relates to undetermined one. Any data flow from "Intuition" to "Logic" will make "Logic" undetermined and entered into "Intuition" component as a result. Any data flow from "Logic" to "Intuition" can change behavior of "Intuition" in undetermined way (because Intuition is undetermined) that most possible will require retraining in case of AI solution. In this case we can say that "Logic" will be entered into "Intuition".

Epstein showed that two systems of human brain are operating simultaneously: experiential/intuitive and rational/ analytic. Both systems are adaptive, but in different ways, and neither system is generally superior to the other as each has unique strengths and limitations [31]. This fact is an additional prove of the model, represented in Fig. 5 and shows that "Intuition" and "Logic" components can be considered as ones originated from "Domain" level of 3-tier architecture.

It should be mentioned that actual trained and error-free AI components can contain a logical part. An evidence of a logical part is a fact that some AI components are determined on the training set (for example overfitting problem). Another example may be concluded in fact that AI unit may be designed as logically joined neural subnets. Thus, the 4component architecture is an idealistic model.

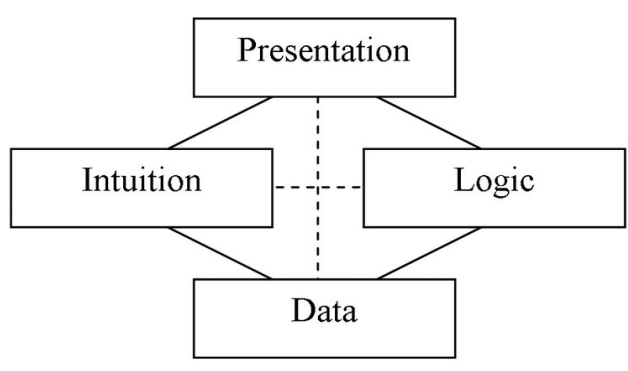

Fig 5. Relations in Four-Component Architecture

In real projects "Intuition" components can be ones where undetermined calculations are performed. This assumption allows considering different intelligent components performing undetermined calculations to be considered as "Intuition" components. Speaking strongly detailed description and requirements aimed to understand which component should be specific code or device should be related. But for this study components performing determined calculations should be considered as belonging to "Logic". Components performing undetermined calculations in their turn should be considered as belonging to "Intuition". Such division allows using the 4-component IS architecture at the beginning stages of design.

The 4-component IS architecture can be presented in more details where "Intuition" is presented as 4 levels (See Fig. 6). This case shows architecture where "Intuition" contains its own data source, logic and presentation serving for integration purposes. An example of such architectures can be a quantum computing unit that needs to transfer data to/ from the quantum gates according to specific logic. Besides all "Intuition" accumulates data during the training, that also can be indicated as a data component and separated as a "gene" (that is useful for genetic algorithms).

\section{B. Underfitting and Overfitting Problems}

Underfitting and overfitting problems could be considered as classic in the ML. The problems are quietly often discussed in literature at different levels - from theory to prac- 


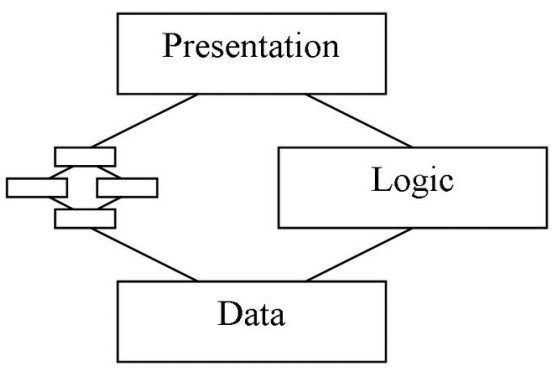

Fig 6. Nesting in Four-Component Architecture

tice [32-36]. These problems can be analyzed through the prism of the 4-component model. AI can be considered here as consisting of all the four components considered. The problems will be discussed at high abstraction level and is presented as a hypothesis. Proof of this hypothesis needs actual experiments that is beyond of the scope of this paper.

The model enables having a new look to the underfitting and overfitting problems in the ML. "Intuition" component relates to the Mythic reality of McWhinney's model that in its turn relates to the free will. "Logic" component relates to the Sensory reality of McWhinney's model that consequently relates to the deterministic realities. These facts allow one to draw the following conclusions:

- Underfitting issues relate to cases where "Intuition" component dominates over "Logic" component;

- Overfitting issues relate to cases where "Logic" component dominates over "Intuition" component.

Dominance of "Intuition" can also be due to dominant data/command flows through "Data" - "Intuition" and/or "Presentation" -"Intuition" links. Dominance of "Logic" in its turn can be due to dominant data/command flows through "Data"-"Logic" and/or "Presentation"-"Logic" links accordingly. Thus, data and interface ("Presentation") can contribute underfitting and overfitting problems. For the Artificial Neural Networks (ANN) "Presentation" can relate to input and output neurons.

Concerning to the modern methods of solving the underfitting and overfitting problems one can say the following. Network reduction allows decreasing AI capability to memorize weak relations in the training dataset that in its turn reduces dominance of Logic. At the same time, it can relate to dominance of the links to "Presentation" and "Data" if reduces input or output neurons (Neighbor and deeper neurons can also have influence. This influence should be studied).

Penalty method, expansion of the training data method, regularization method relate to "Data". Regularization method can relate also to "Presentation". Early stopping method prevents growth of AI dominance over Logic by preventing strengthening one part over another.
Techniques against the underfitting includes expansion of training dataset ("Data"), increase the number or size of parameters in the model ("Data", "Presentation"), increase the complexity of the model ("AI", "Logic"), increase the training time (strengthening "Logic").

\section{AI Threat Problem}

There is a concern that AI can get out of human control and significantly harm humanity. Implementation of technological inventions without an in-depth laboratory analysis of the consequences is real precondition for this threat [37]. Analyses of AI threat with means of the 4-component model leads to considering the problem sourced in two spheres:

- Logical - in case if AI system trained to do harm the people;

- Intuition - in case if AI system not trained not to harm people.

Although it is just a hypothesis, it shows another possible application of the 4-component model.

\section{Value alignment problem}

The 4-component architecture can help solve the value alignment problem of aligning AI values with humans. McWhinney's model can be used as a mediator between human values and the 4-component architecture. Description of human values in terms of McWhinney's model is psychologists' problem. Nevertheless, alignment of described human values with the 4-component architecture is problem of programmers. Because 3 of 4 components of the architecture are well known, studied, and used in practice, while a new component "Machine Intuition" is the only subject for such research.

It should be mentioned that the value alignment problem should be considered not only for AI, ANN, QC solution, but also for classical solutions. One example of aligning values with humans is a user interface, that relates to visual aesthetics experience. Another example is the software quality (there are many quality assurance methodologies that could be helpful).

\section{E. Business Processes Modeling and Moral Decision Making}

Business Processes Modeling (BPM) widely uses diagrams and formulae. It shows its orientation to strong logic and determined calculations. Intuition in classic BPM is the prerogative of a human. As this research shows, complex systems and AI introduce new "Intuition" component which is used more and more in IS. So, BPM can include (or may be separate to specific technique) modeling of intuitive processes. These processes can be based on experience [31].

Miller selected success groups and success factors in moral decision making and algorithms [38]. But it should be mentioned that morality of the decision is based on evaluation of consequences of the decision and originally this evaluation can be made only by human. As a result automatic evaluation of morality by the computer can be only based on 
experience in similar cases and corresponds to "Intuition" component of the architecture.

\section{F. Sustainable Development and Smart Cities}

There are four dimensions of Sustainable Development (SD), i.e. ecological, economic, socio-cultural, and political [39]. These dimensions introduce behavior with some non determinism. AI solutions are very useful to handle issues with such behavior. Presented 4-component architecture seems helpful to analyze SD issues and develop solutions.

Smart Culture is the specific component of Smart Cities. Current understanding of Smart Culture is concluded in provision of information [40]. AI can handle issues in culture, related to non deterministic calculations, such as assessment of music, paintings, and other artifacts. The 4-component architecture can help to develop such solutions.

\section{Conclusion}

AI and QC introduce new kind of IS where calculations significantly differ on classical ones. These calculations contain some kind of uncertainty and non-determinism and introduce new problems. The 4-component model is a simple tool allowing design, develop, and analyze of such IS. Moreover, it allows better understanding and learning these systems and solve related problems. Application of the 4component model to Smart City and related issues seem to be very promising. There is also interest to use the model for quality assurance and evaluation of ability of IS use in critical systems.

Presentation of the 4-component AI architecture in lectures "Programming technology" is approved by Baku State University. It is implicit proof of interest to the model and chance to discuss it with the students.

A "Machine Intuition" component is the point where software developers and psychologists can cooperate to create human friendly solutions. Such cooperation could also be helpful to better understand the human psyche.

It is also hypothesis that growing impact of non-deterministic behavior will prevent growth of complexity and functionality of IS. In other words, a "Machine Intuition" component should not remain without attention of researchers.

\section{A. Disadvantages and Further Research}

Disadvantages of the 4-component IS architecture are concluded in fact that there is no obvious boundary between Intuition and Logic components. Often even classical software has bugs (it could be acceptable levels of bugs) introducing some kind of uncertainty and non-determinism. But determination of "boundary" between solid logic and uncertainty is a difficult task. This makes the 4-component IS architecture more theoretical, rather than a practical tool.

There is huge interest to study Quality Assurance methodologies with application to 4-component architecture. Integration of psychological approaches could improve quality of complex IS.
There is also the fact that the advantages of the architecture have not been practically proved yet. Further research of the 4-component IS architecture can include:

- proof of benefits of the model in practical use including teaching of students;

- a deeper and wider research of the model;

- the way the model can help solve underfitting and overfitting problems;

- the way the model can help solve value alignment problem;

- possibility of integration with programming languages and code constructions;

- possibility of use as a design pattern in IS development;

- application in Quantum Computing;

- IS quality assurance;

- application in Business Process Modeling;

- SD, Smart Cities, Smart Culture;

- AI threat problem.

It should be mentioned that the discussion was carried out for AI. QC is now considered sufficient. There is an assumption that the model is useful for QC as well. Although $\mathrm{AI}$ is a young conception, QC is much younger. Further development of QC can require revision of abilities of 4-component model to apply to $\mathrm{QC}$ and implement addition research.

\section{ACKNOWLEDGMENT}

I wish to express my sincere gratitude to SINAM Ltd. and Baku State University for their kind support and inspiration in the research.

\section{REFERENCES}

[1] S. Fulton, "What is quantum computing today? The how, why, and when of a paradigm shift", 2020, https://www.zdnet.com/article/whatis-quantum-computing-understanding-the-how-why-and-when-ofquantum-computers/, accessed on 19th April 2021.

[2] L. Ng, "The AI Wolf That Preferred Suicide Over Eating Sheep", https://onezero.medium.com/the-ai-wolf-that-preferred-suicide-overeating-sheep-49edced3c710, accessed in July, 2021.

[3] J. Lehman, J. Clune, D. Misevic, C. Adami, L. Altenberg, J. Beaulieu, P. Bentley, S. Bernard, G. Beslon, D. Bryson, N. Cheney, P. Chrabaszcz, A. Cully, S. Doncieux, F. Dyer, K. Ellefsen, R. Feldt, S. Fischer, S. Forrest, A. Fŕenoy, C. Gagńe, L. Goff, L. Grabowski, B. Hodjat, F. Hutter, L. Keller, C. Knibbe, P. Krcah, R. Lenski, H. Lipson, R. MacCurdy, C. Maestre, R. Miikkulainen, S. Mitri, D. Moriarty, J. Mouret, A. Nguyen, C. Ofria, M. Parizeau, D. Parsons, R. Pennock, W. Punch, T. Ray, M. Schoenauer, E. Schulte, K. Sims, K. Stanley, F. Taddei, D. Tarapore, S. Thibault, R. Watson, W. Weimer, J. Yosinski, "The Surprising Creativity of Digital Evolution: A Collection of Anecdotes from the Evolutionary Computation and Artificial Life Research Communities", Artif Life, 26 (2), pp. 274 306, 2020, https://doi.org/10.1162/artl_a_00319.

[4] L. Yaeger, "Computational genetics, physiology, metabolism, neural systems, learning, vision, and behavior or PolyWorld: Life in a new context", Artificial Life III, vol. XVII, pp. 263-298, Addison-Wesley, 1993, https://doi.org/10.1.1.38.6719.

[5] R. Feldt, "Generating multiple diverse software versions with genetic programming", Proc. 24th EUROMICRO Conference, vol.1 pp. 387394, 1998, https://doi.org/10.1109/EURMIC.1998.711831.

[6] R. Cook, "How Complex Systems Fail", Cognitive Technologies Laboratory, University of Chicago, Chicago, IL, USA, 1998. 
[7] R. Williams, R. Yampolskiy, "Understanding and Avoiding AI Failures: A Practical Guide", Philosophies, 6(3):53, 2021, https://doi.org/10.3390/philosophies6030053.

[8] S. McGregor, C. Custis, J. Yang, J. McHorse, S. Reid, S. McGregor, S. Yoon, C Olsson, R. Yampolskiy, "AI Incident Database", 2021, https://incidentdatabase.ai/, accessed in July, 2021.

[9] P. Scott, R. Yampolskiy, "Classification Schemas for Artificial Intelligence Failures", Delphi - Interdisciplinary Review of Emerging Technologies, vol. 2, iss. 4, pp. 186-199, 2019, https://doi.org/10.21552/delphi/2019/4/8.

[10] K. Lui, J. Karmiol, "AI Infrastructure Reference Architecture”, IBM Systems, 2018

[11] K. Bertels, A. Sarkar, T. Hubregtsen, M. Serrao, A. A. Mouedenne, A. Yadav, A. Krol, I. Ashraf, "Quantum computer architecture: towards full-stack quantum accelerators", Design, Automation and Test in Europe, pp. 1-6, 2020, https://doi.org/10.23919/DATE48585.2020 9116502.

[12] N. Jones, R. Meter, A. Fowler, P. McMahon, J. Kim, T. Ladd, Y. Yamamoto, "Layered Architecture for Quantum Computing", Phys. Rev. X 2, 031007, 2012, https://doi.org/10.1103/PhysRevX.2.031007.

[13] P. Berander, L. Damm, J. Eriksson, T. Gorschek, K. Henningsson, P. Jönsson, S. Kågström, D. Milicic, F. Mårtensson, K. Rönkkö, P. Tomaszewski, "Software quality attributes and trade-offs", Blekinge Institute of Technology, June 2005.

[14] S. Wagner, K. Lochmann, S. Winter, A. Goeb, M. Klaes, "Quality Models in Practice: A Preliminary Analysis", ESEM'09, 2009.

[15] S. Wagner, K. Lochmann, L. Heinemann, M. Kläs, A. Trendowicz, R. Plösch, A. Seidl, A. Goeb, J. Streit, "The Quamoco Product Quality Modelling and Assessment Approach", 34th Int. Conf. on Software Engineering (ICSE), pp. 1133-1142, 2012 https://doi.org/10.1109/ICSE.2012.6227106.

[16] S. Wagner, K. Lochmann, S. Winter, A. Goeb, M. Kläs, S. Nunnenmacher, "Software Quality Models in Practice. Survey Results", http://mediatum.ub.tum.de/doc/1110601/274701.pdf, accessed in July, 2021.

[17] Wikipedia, the free encyclopedia, http://en.wikipedia.org, accessed in April 2021.

[18] A. Tafti, S. Janosepah, N. Modiri, A. Noudeh, H. Alizadeh, "Development of a Framework for Applying ASYCUDA System with N-Tier Application Architecture", Comm. in Comp. and Inf. Science, vol. 181 pp. 533-541, 2011, https://doi.org/10.1007/978-3-642-22203046.

[19] Z. Durdik, "Architectural Design Decision Documentation through Reuse of Design Patterns", KIT Scientific Publishing, 2016, https://doi.org/10.5445/KSP/1000043807.

[20] M. Fowler, "Patterns of Enterprise Application Architecture", Addison-Wesley, 2011

[21] D. Brée, "Artificial Intelligence and Cognitive Psychology: A New Look at Human Factors", Human-Computer Interaction, 1988, https://doi.org/10.1007/978-3-642-73402-1_17.

[22] T. Moran, S. Card, "Applying Cognitive Psychology to Computer Systems", A Graduate Seminar in Psychology, 1980.

[23] A. E. Bolock, J. Salah, Y. Abdelrahman, C. Herbert, S. Abdennadher, "Character Computing: Computer Science meets Psychology", MUM'18, pp. 557-562, 2018, https://doi.org/10.1145 3282894.3286060
[24] P. Panditi, "Psychology - The Land That Software Engineering Forgot", Proc. of Innovations in Software Engineering Conference, 2018, https://doi.org/10.1145/3172871.3172889.

[25] K. VAT, "Teaching Software Psychology: Expanding the Perspective", Proc. of the 31st SIGCSE Technical Symposium on Comp. Sci. Ed., 2000, https://doi.org/10.1145/331795.331892.

[26] P. Young, "Understanding NLP Principles \& Practice", Crown House Publishing 2nd ed, 2004.

[27] С. Ковалёв, "Психотерапия человеческой жизни”, Москва, 2018.

[28] W. McWhinney, "Growing Into the Canopy", Journal of Transformative Education, vol. 5 no. 3 pp. 206-220, 2007, https://doi.org/10.1177/1541344607307023.

[29] W. McWhinney, J. Webber, D. Smith, B. Novokowsky, "Creating Paths of Change: Managing Issues and Resolving Problems in Organizations", SAGE Publications, 1997.

[30] N. Walliman, "Research Methods. The Basics", Routledge, London, 2011.

[31] S. Epstein, "Demystifying Intuition: What it is, What it Does, and How it Does it", Psychological Inquiry, 21, 295-312, 2010.

[32] H. Zhang, L. Zhang, Y. Jiang, "Overfitting and Underfitting Analysis for Deep Learning Based End-to-end Communication Systems", 11th Int. Conf. on Wireless Comm. and Sign. Processing (WCSP), pp. 1-6, 2019, https://doi.org/10.1109/WCSP.2019.8927876.

[33] H. Gabbar, R. Khan, "Methods to avoid over-fitting and under-fitting in supervised machine learning (comparative study)", CompSci Comm. Instr. Dev., pp. 163-172, 2014.

[34] F. Yusufi, A. Ahmed, J. Ahmad, "Modelling and developing diabetic retinopathy risk scores on Indian type 2 diabetes patients", Int. J. Diabetes Dev Ctries 39, pp. 29-38, 2019, https://doi.org/10.1007/s13410-018-0652-z.

[35] E.A. Martínez-García, N. Rodríguez, R. Rodríguez-Jorge, J. MizeraPietraszko, J. Sheba, R. Mohan, E. Magid, "Non Linear Fitting Methods for Machine Learning", Lecture Notes on Data Engineering and Communications Technologies, vol 13 pp. 807-818, 2018, https://doi.org/10.1007/978-3-319-69835-9 76.

[36] X. Ying, "An Overview of Overfitting and its Solutions", Journal of Physics: Conference Series, vol. 1168, issue 2, 2019 , https://doi.org/10.1088/1742-6596/1168/2/022022.

[37] M. Tegmark, "An Open Letter: Research Priorities for Robust and Beneficial Artificial Intelligence, https://futureoflife.org/ai-open-letter. accessed in April 2021.

[38] G. Miller, "Artificial Intelligence Project Success Factors: Moral Decision-Making with Algorithms", Proceedings of the 16th Conference on Computer Science and Intelligence Systems, M. Ganzha, L. Maciaszek, M. Paprzycki, D. Ślęzak (eds). ACSIS, Vol. 25, pp. 379-390, 2021, http://dx.doi.org/10.15439/2021F26

[39] E. Ziemba, "The ICT adoption in enterprises in the context of the sustainable information society", Proceedings of the Federated Conference on Computer Science and Information Systems, vol. 11 pp. 1031-1038, 2017, https://doi.org/10.15439/2017F89.

[40] M. Fanea-Ivanovici, M. Pană, "From Culture to Smart Culture. How Digital Transformations Enhance Citizens' Well-Being Through Better Cultural Accessibility and Inclusion", IEEE Access, vol. 8, pp. 37988-38000, 2020, https://doi.org/10.1109/ACCESS.2020.2975542. 